\title{
Shape-Anisotropy-Induced Ordered Packings in Cylindrical Confinement
}

\author{
Weiwei Jin (金炜炜)๑, ${ }^{*}$ Ho-Kei Chan (陈浩基)๑, ${ }^{\dagger}$ and Zheng Zhong (仲政) \\ School of Science, Harbin Institute of Technology (Shenzhen), Shenzhen 518055, China
}

(Received 10 July 2019; revised manuscript received 29 April 2020; accepted 26 May 2020; published 16 June 2020)

\begin{abstract}
Densest possible packings of identical spheroids in cylindrical confinement have been obtained through Monte Carlo simulations. By varying the shape anisotropy of spheroids and also the cylinder-to-spheroid size ratio, a variety of densest possible crystalline structures have been discovered, including achiral structures with specific orientations of particles and chiral helical structures with rotating orientations of particles. Our findings reveal a transition between confinement-induced chiral ordering and shapeanisotropy-induced orientational ordering and would serve as a guide for the fabrication of crystalline wires using anisotropic particles.
\end{abstract}

DOI: 10.1103/PhysRevLett.124.248002

Physicists and mathematicians have long been fascinated by problems of particle packing. Dense packings of hard particles have been widely used as models for understanding the structural properties of condensed matter systems such as liquids, glasses, crystals, heterogeneous materials, and granular media [1-4]. Of such dense-packed systems, packings of hard spheres have been studied most extensively thanks to their geometric simplicity and richness in thermodynamic behavior. In recent years, packings in confinement have become a subject of immense interest, as they are relevant to many different entities and applications such as cell morphology within epithelial tissues [5], phyllotaxis in plants like sunflower seeds and pine cones [6], droplet-based fabrication of microparticles [7], and drug delivery [8], as well as a number of self-assembled systems in cylindrical confinement [9-20].

Previous experimental [9-20] and computational [15, 21-28] studies showed that the densest possible packings of identical hard spheres within a cylinder correspond to a geometric frustration by the curved cylindrical surface, resulting in distinct helical configurations (e.g., single, double, and triple helices) at specific ranges of the diameter ratio $\mathcal{D}$ between the cylinder and the spheres. At $\mathcal{D} \leq 2.71486$, all spheres are in contact with the confining cylinder, in which case it is possible to adopt a line-slip description for the continuous transition between different helical structures [25]. Densest possible packings in such quasi-1D confinement can generally be referred to as columnar crystals, as they are periodic along the axial direction of the cylinder with every unit cell being a rotation (of twist angle $\alpha$ ) of the previous one.

Published by the American Physical Society under the terms of the Creative Commons Attribution 4.0 International license. Further distribution of this work must maintain attribution to the author(s) and the published article's title, journal citation, and DOI.
It has been found that the chirality of such columnar crystals can lead to novel optical [19] or electrical [20] properties.

Compared to their spherical counterparts, much less research has been conducted on the packings of anisotropic particles in confinement. Such packings involve particle orientations as additional degrees of freedom, and hence they exhibit a much greater variety of self-assembled structures $[29,30]$. Here we regard spheroids (ellipsoids of revolution) as the simplest extensions of spheres to include orientational degrees of freedom, where the shape anisotropy of a spheroid can simply be described in terms of its aspect ratio.

The optimal packings of a system of hard particles at high pressures are intimately related to the thermodynamic phases of the system [31]. For example, the FrenkelMulder phase diagram of spheroids [32,33], which serves as a basis for studies of nematic phases and isotropicnematic transitions [34,35], has been updated [36,37] as a result of discoveries of densest possible crystal structures of ellipsoids [38]. Similarly, a square-triangle crystal family has been included in an updated phase diagram of biaxial ellipsoids [39]. It is therefore believed that the present study of densest possible packings of spheroids in cylindrical confinement could lead to discoveries of novel ordered phases.

In this Letter, we demonstrate that complex structures with rotating or specific orientations of spheroids can be obtained through self-assembly of spheroids in cylindrical confinement. We report our findings on the unexplored problem of densest possible packings of identical spheroids in a cylinder of diameter $D_{c}$. For identical spheres packed in a cylinder, the densest possible configurations are determined only by the diameter ratio $\mathcal{D}$. But for packings of spheroids, the densest possible configurations are also determined by the aspect ratio of particles. Our work provides physical insights into how confinement conditions and particle anisotropy together determine the optimal 
packings of a system. The results would serve as a guide for the fabrication of functional ordered structures such as colloidal crystal wires [11] through template-based selfassembly [17-20].

We employed the Monte Carlo (MC) method adapted from the adaptive shrinking cell optimization scheme $[39,40]$ to explore densest possible packings of identical hard spheroids confined in cylinders (see Supplemental Material [41] for details). Let $a$ and $b=c$ be the axis lengths of a spheroid in general, with the conditions $a=b$, $a>b$, and $a<b$ defined for the specific cases of a sphere, a prolate, and an oblate, respectively. The aspect ratios $\mathcal{A} \equiv$ $\max (a, b) / \min (a, b)$ of these specific cases are given by $\mathcal{A}=1, \mathcal{A}=a / b$, and $\mathcal{A}=b / a$, respectively. For any spheroid packed inside a cylinder of diameter $D_{c}$, the inequality $D_{c} \geq b$ must be satisfied. The size ratio $\mathcal{D} \equiv$ $D_{c} / b \geq 1$ is therefore used as a universal parameter to describe the effect of cylindrical confinement. Our investigation focused on prolates within $\mathcal{A} \in(1,3]$ and oblates within $\mathcal{A} \in(1,1.2]$ for $\mathcal{D} \in(1,2)$. The chosen parameter range of $\mathcal{D}$ corresponds to the existence of three densest possible structures of identical spheres, i.e., zigzag, single helix, and double helix, appearing sequentially for increasing $\mathcal{D}$ with structural transitions at $\mathcal{D}=1+\sqrt{3} / 2$ and $1+$ $4 \sqrt{3} / 7[22,43]$. Within the chosen ranges of $\mathcal{A}$, the densest possible structures could be obtained within reasonable computational times, and the number of densest possible structures discovered in the oblate regime is comparable to that in the prolate regime. For each pair of parameter values $\{\mathcal{A}, \mathcal{D}\}$, we generated dense packings of $N=1$ to $N=24$ spheroids via $\mathrm{MC}$ simulations. Possible packings obtained from simulated $\mathrm{MC}$ compressions were then used as a guide for constructing corresponding crystal structures, where a sequential deposition approach $[23,26,44]$ was adopted. By varying both parameters $\mathcal{A}$ and $\mathcal{D}$, we observed a transition between chiral ordering induced by the cylindrical confinement and orientational ordering induced by the shape anisotropy of particles.

Seven types of densest possible columnar crystalsnamely, single helix, double helix, triple helix, unidirectional zigzag (uni-zigzag), bi-directional crystal (bi-crystal), achiral doublets [22], and achiral triplets [22] —have been discovered via MC simulations (see Fig. 1). The orientation of each spheroid, as depicted by an arrow in Fig. 1 , is the vector $\vec{n}$ defined in Fig. 2(a). The single-helix structure is further classified into two subtypes: single helix + and single helix -. Let the particles be indexed in ascending order of their vertical positions and be positioned in a right-handed manner along the positive direction of the $Z$ axis. A single helix with rotating orientations of particles is classified as a "+" ("-") subtype if $\left(\vec{r}_{i} \times \vec{p}_{i}\right) \cdot \hat{z}>0(<0)$, where $\vec{p}_{i}$ is the projected direction of spheroid $i$ and $\vec{r}_{i}$ denotes the vector from the center of the projected cylinder to the particle center [see Fig. 2(b)]. For each spheroid, there exists only one possible direction of $\vec{p}_{i}$ except for the $\theta=90^{\circ}$ case because

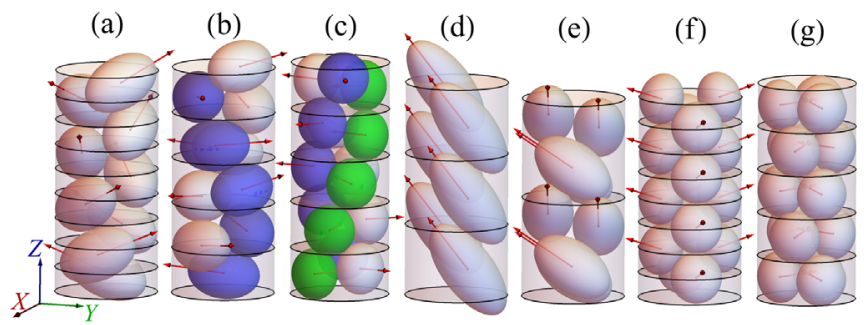

FIG. 1. Seven types of crystal structures discovered through MC simulations. (a) single helix, (b) double helix, (c) triple helix, (d) uni-zigzag, (e) bi-crystal, (f) achiral doublets, and (g) achiral triplets with one, two, three, two, four, two, and three particles in their unit cells, respectively.

the polar angle $\theta$ is defined only for the range of $\left[0,90^{\circ}\right]$. This classification for the single-helix structure takes into account both the positional and the orientational order of the particles.

Figure 3(a) shows a map of densest possible structures with 12 distinct regions, with three of them spanning the regimes of oblates and prolates. For the other nine regions, five of them are located in the oblate regime, and four are located in the prolate regime. The oblate regime involves six of the seven columnar structures described above, while the prolate regime involves only four. Although the chosen range of $\mathcal{A}$ for oblates is much smaller than that for prolates, the oblate regime already displays a greater number of distinct regions and a greater structural variety than the prolate regime. Figure 3(b) displays the packing densities of the densest possible structures shown in Fig. 3(a). At boundaries between different structures, the packing density varies continuously but in a nonsmooth manner, except for the smooth variation at each single-to-doublehelix transition. The high degree of oblate-prolate asymmetry in both Figs. 3(a) and 3(b) arises from the difference in geometry between prolates $\left(D_{c} / a=\mathcal{D} / \mathcal{A}<\mathcal{D}\right)$ and oblates $\left(D_{c} / a=\mathcal{D} \mathcal{A}>\mathcal{D}\right)$ with respect to the confining cylinder. Such asymmetry is expected to exist for the corresponding diagram of thermodynamic phases, as crystal structures of maximum densities should all be included in the phase diagram $[36,37]$. This is in contrast to cases of bulk packings, where the maximal achievable density at any given aspect
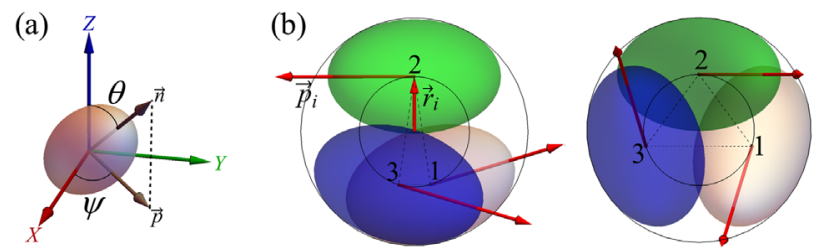

FIG. 2. (a) The orientation of a prolate (an oblate) is defined as the direction $\vec{n}$ of the longest (shortest) axis, with $\theta \in\left[0,90^{\circ}\right]$ defined as the polar angle of the particle and $\psi \in\left[0,360^{\circ}\right]$ as the azimuthal angle between the projection $\vec{p}$ and the $X$ axis. (b) Configurations of the two subtypes of single helices, "+" (left) and "-" (right). 

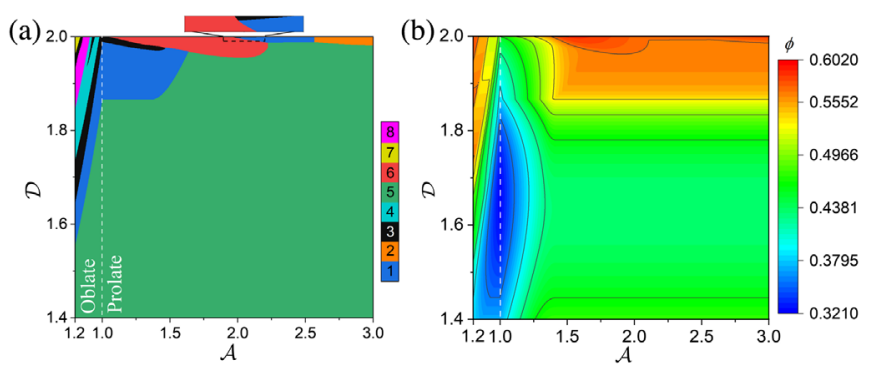

FIG. 3. (a) Twelve regions from the eight types/subtypes of densest possible crystal structures: single helix $+(1)$, single helix - (2), double helix (3), triple helix (4), uni-zigzag (5), bi-crystal (6), achiral doublets (7), and achiral triplets (8), where the uni-zigzag region extends towards $\mathcal{D}=1$ for the whole range of $\mathcal{A}$. The enlarged region consists of a double-helix region in the vicinity of $\mathcal{D}=2$. (b) Packing densities of densest possible structures as a function of $\mathcal{A}$ and $\mathcal{D}$.

ratio is the same for both oblates and prolates [38] and where the corresponding phase diagram is practically oblate-prolate symmetric [36,37].

For a spheroid, the polar angle $\theta$ and the azimuthal angle $\psi$ are used to characterize the orientation of the particle, as illustrated in Fig. 2(a). The packing density $\phi$ of a single helix at given values of $\mathcal{A}$ and $\mathcal{D}$ is only a function of the polar angle $\theta$ and the twist angle $\alpha$. The densest possible single-helix structure was obtained through a binary search of $\theta$ and $\alpha$. Figure 4(a) shows the packing density of confined prolates as a function of $\theta$ for various values of $\mathcal{A}$, where for each value of $\theta$ the packing density has already been maximized with respect to the twist angle $\alpha$. Similar results have been obtained for the packings of oblates. At $\theta=0^{\circ}$, where all spheroids are aligned along the cylinder's
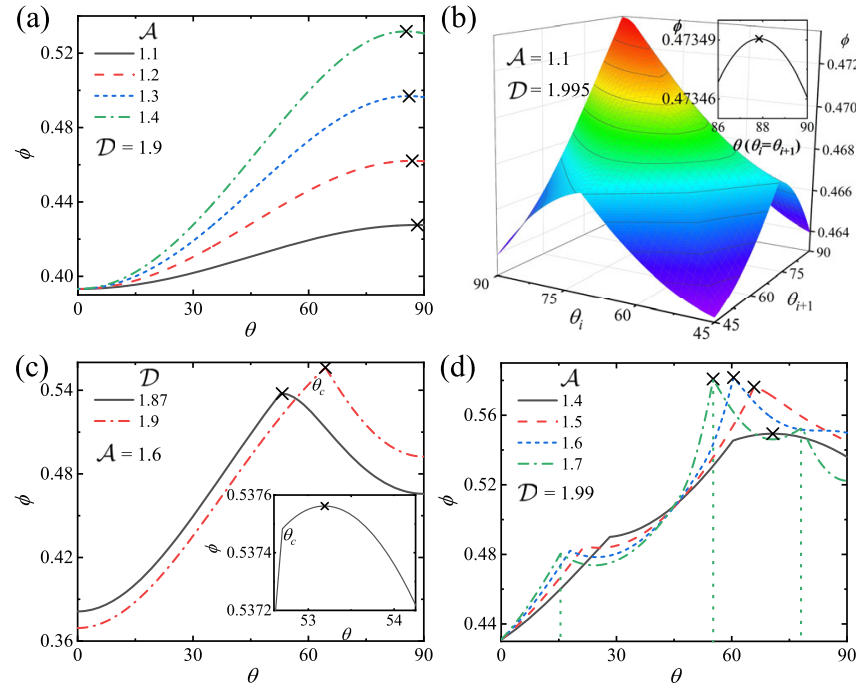

FIG. 4. The packing density $\phi$ of confined prolates as a function of $\theta$ for (a) the single helix, (b) the double helix, (c) the uni-zigzag, and (d) the bi-crystal at various values of $\mathcal{A}$ and $\mathcal{D}$. The maximum point of each curve is marked with a cross. axis, all four cases have the same lowest packing density because the dense-packed structure of each case is a simple affine transformation of the densest possible single-helix structure for sphere packing. The densest possible singlehelix structures of spheroids appear at some nonzero values of $\theta$, indicating that the system's density is maximized via the particles' rotational degrees of freedom.

Let $\Delta \psi_{i, i+1}=\psi_{i}-\psi_{i+1}$ be the azimuthal angle difference between two successive particles. For the double-helix structure, we have

$$
\Delta \psi_{i, i+1}=\Delta \psi_{i+2, i+3} \neq \Delta \psi_{i+1, i+2},
$$

where the $(i+2)$ th and $(i+3)$ th particles are replicas of the $i$ th and $(i+1)$ th particles in the subsequent unit cell, respectively. At each pair of given polar angles $\theta_{i}$ and $\theta_{i+1}$, the packing density is maximized with respect to the azimuthal angle differences $\Delta \psi_{i, i+1}$ and $\Delta \psi_{i+1, i+2}$. The packing density was then computed as a function of $\theta_{i}$ and $\theta_{i+1}$, as shown in Fig. 4(b). The maximum packing density appears at a point of $\theta_{i}=\theta_{i+1}$ as the plot surface is symmetric with respect to $\theta_{i}$ and $\theta_{i+1}$. Hence, any pair of successive spheroids in the densest possible double helix always share the same polar angle $\theta=\theta_{i}=\theta_{i+1}$. Plots of the packing density as a function of $\theta$ are similar to plots for the single-helix structure with a single peak appearing at $\theta \in\left[0,90^{\circ}\right]$ as shown in the inset of Fig. 4(b).

The achiral uni-zigzag crystal has a unit cell that contains two spheroids aligned in the same direction. The twist angle $\alpha$ between successive unit cells is zero, and the spheroid centers are arranged in the manner of a planar-zigzag structure. For prolates (see Supplemental Material [41] for a discussion of oblates), consider the critical aspect ratio

$$
\mathcal{A}_{p}=\frac{\sqrt{1+4 \mathcal{D}^{2}+\sqrt{1-56 \mathcal{D}^{2}+16 \mathcal{D}^{4}}}}{2 \sqrt{2}}
$$

for a prolate at $\mathcal{D}>1+\sqrt{3} / 2$. At $\mathcal{A}<\mathcal{A}_{p}$, the packing density of the densest possible single-helix structure is higher than that of any uni-zigzag structure, so that only uni-zigzags at $\mathcal{A} \geq \mathcal{A}_{p}$ could be considered as candidates of densest possible structures. Figure 4(c) shows the packing density $\phi$ as a piecewise function of $\theta$ with a transition point at $\theta_{c}$. Each prolate of a unit cell is in contact with its counterparts in adjacent unit cells only at $\theta \leq \theta_{c}$ but not at $\theta>\theta_{c}$. The maximum packing density appears at $\theta=\theta_{c}$ if $\mathcal{D} \in[\sqrt{7 / 2}, 2)$, while for cases of $\mathcal{D} \in(1+\sqrt{3} / 2, \sqrt{7 / 2})$, the maximum density occurs at a polar angle of $\theta_{m}\left(\theta_{m}>\theta_{c}\right.$, see Supplemental Material [41]). At $\mathcal{D} \in(1,1+\sqrt{3} / 2]$, the uni-zigzag structure of prolates populates the whole range of $\mathcal{A}$. At $\mathcal{A} \leq \sqrt{\mathcal{D}}$, each prolate with $\theta=90^{\circ}$ in the cell is in contact with the cylindrical wall, with one end of the particle's short axis being the point of contact. This structure can be obtained by simply stretching confined spheres 
along the direction perpendicular to the zigzag plane. The picture at $\mathcal{D} \in(1,1+\sqrt{3} / 2]$ and $\mathcal{A}>\sqrt{\mathcal{D}}$ is similar to that at $\mathcal{D} \in(1+\sqrt{3} / 2, \sqrt{7 / 2})$ and $\mathcal{A} \geq \mathcal{A}_{p}$ (see Supplemental Material [41] for analytic expressions of the packing density). It follows that the packing densities of the densest possible uni-zigzag structures at $\mathcal{A} \geq \sqrt{2}$ are independent of $\mathcal{A}$ and take on a constant value at any given value of $\mathcal{D}$. The reason is that any increase in the packing density due to an increase in $\mathcal{A}$ is counteracted by a decrease in the packing density due to an increase in the height of the unit cell.

The achiral bi-crystal (twist angle $\alpha=0$ ) has a unit cell that consists of two pairs of prolates, with a unique orientation for each pair. The prolates in each of such pairs follow the same arrangement as that of two successive particles in the uni-zigzag structure. Each pair of prolates is a rotated mirror image of its neighboring pairs, with the $X Y$ plane being the plane of reflection and the $Z$ axis being the axis of rotation. It follows that all particles in this crystal structure share the same polar angle $\theta$, where, at $\theta=0^{\circ}$, the structure is a simple affine transformation along the $Z$ axis such that the packing density at any given value of $\mathcal{D}$ is the same for all values of $\mathcal{A}$ [see Fig. 4(d)]. On the other hand, a densest possible structure is not necessarily a structure with the maximum number of contacts. As shown in Fig. 4(d), the densest possible structure exhibits the maximum number of contacts at $\mathcal{A}=1.5,1.6$, and 1.7 but not at $\mathcal{A}=1.4$.

For all the seven structures discovered, the spheroids within any densest possible structure are all in contact with the cylindrical wall. At any given polar angle $\theta$, the centers of the cylinder-touching spheroids are located at shortest distances from the cylindrical boundary such that they all lie on the same inner cylindrical surface. This applies also to the particular case of sphere packing where, in the absence of shape anisotropy of particles, any cylindertouching sphere automatically has its center located closest to the cylindrical boundary. For all other structures except the triple-helix structure, all particles within any densest possible structure share the same value of $\theta$. For the triplehelix structure, there are a total of three oblates in a unit cell, with at least two of them sharing the same value of $\theta$.

Any transition from one type of densest possible crystal to another is described as either structurally continuous or discontinuous. In the continuous case, the two types of crystals at the transition point are structurally the same. In the discontinuous case, the transition point corresponds to a packing density shared by two different structures. With reference to Fig. 3(a), the continuous single helix to unizigzag transitions at $\mathcal{A} \in[(1+\sqrt{3}) / 2,1.636)$ and $\mathcal{D} \in$ $(1+\sqrt{3} / 2,1.968)$ can be understood in terms of a transition between two types of ordering. Figure 5 displays the polar angle $\theta$ of densest possible packings of prolates as a function of the aspect ratio $\mathcal{A}$ for five different values of $\mathcal{D}$, where the isotropic limit $\mathcal{A} \rightarrow 1$ corresponds to

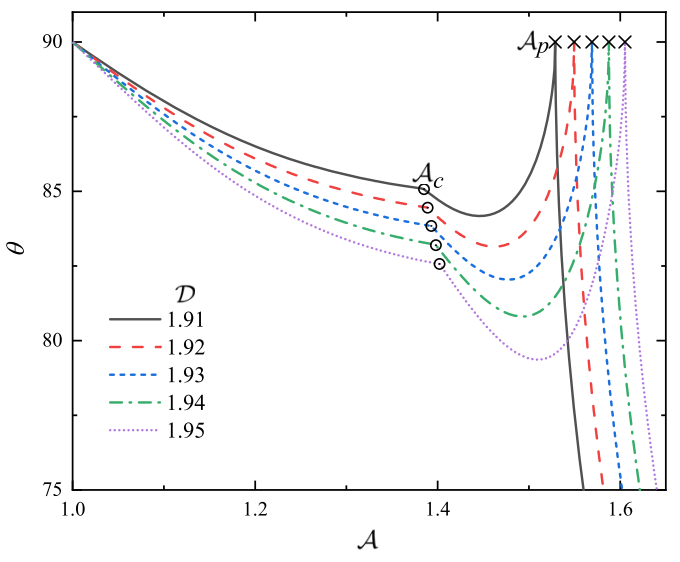

FIG. 5. Plot of the polar angle $\theta$ of densest possible structures of prolates as a function of $\mathcal{A}$. For each curve, the single helix and the uni-zigzag correspond respectively to the regimes $\mathcal{A}<\mathcal{A}_{p}$ and $\mathcal{A} \geq \mathcal{A}_{p}$. The critical values $\mathcal{A}_{c}$ and $\mathcal{A}_{p}$ are marked with circles and crosses, respectively.

helical packings of spheres. For each curve, there exist two critical values of $\mathcal{A}$, denoted as $\mathcal{A}_{c}$ and $\mathcal{A}_{p}$, respectively, with each critical value corresponding to a discontinuity in the first derivative of the curve. At $\mathcal{A} \leq \mathcal{A}_{c}$, the densest possible structure is a single helix with only a single contact point between each prolate and the cylindrical wall. At $\mathcal{A}_{c}<\mathcal{A}<\mathcal{A}_{p}$, the densest possible structure is still a single helix but there exist two contact points between each prolate and the cylinder. At $\mathcal{A} \geq \mathcal{A}_{p}$, the densest possible structure becomes a uni-zigzag with the same orientation for all prolates. This represents a transition from chiral ordering with a spatial rotation of particle orientations to orientational ordering with a uniform orientation shared by all particles. In the former case, the cylinder-touching prolates are packed in a way that their orientations rotate about the $Z$ axis of the cylinder. In the latter case, the particles are elongated to an extent that, within the cylindrical confinement, their orientations are constrained to a single direction only.

The discovery of seven distinct structures in a relatively narrow regime of particle anisotropy suggests that these structures are just the tip of an iceberg, with many more awaiting to be discovered. Future work should include (i) an extension of the map of densest possible structures to wider ranges of $\mathcal{A}$ and $\mathcal{D}$, (ii) a study of the mechanism of continuous transitions between single- and double-helix structures, (iii) a study of the phase diagram of cylindrically confined spheroids at finite pressures, and (iv) an application of the present results to synthesize colloidal crystal wires [11] of spheroidal particles [45,46].

This work was supported by the National Natural Science Foundation of China (Grant No. 11932005), as well as the Science, Technology and Innovation Commission of Shenzhen Municipality (Grants No. JCYJ20160531193515801 and No. ED11409002). W. J. is supported by a project funded by the China 
Postdoctoral Science Foundation (Grant No. 2019M 651273). H.-K. C. is grateful to Professor Denis Weaire, Professor Stefan Hutzler, and Dr. Adil Mughal for bringing him into the field of packing problems during his previous employment as a post-doctoral research fellow at Trinity College Dublin.

*Corresponding author. microwei.jin@gmail.com ${ }^{\dagger}$ Corresponding author. epkeiyeah@yahoo.com.hk

[1] R. Zallen, The Physics of Amorphous Solids (Wiley VCH, Germany, 2004)

[2] S. Torquato, Random Heterogeneous Materials: Microstructure and Macroscopic Properties (Springer Science \& Business Media, New York, 2013), Vol. 16.

[3] P. M. Chaikin, T. C. Lubensky, and T. A. Witten, Principles of Condensed Matter Physics (Cambridge University Press, Cambridge, England, 1995), Vol. 1.

[4] S. V. Franklin and M. D. Shattuck, Handbook of Granular Materials (CRC Press, Boca Raton, 2015).

[5] J.-F. Rupprecht, K. H. Ong, J. Yin, A. Huang, H.-H.-Q. Dinh, A. P. Singh, S. Zhang, W. Yu, and T. E. Saunders, Mol. Biol. Cell 28, 3582 (2017).

[6] S. Douady and Y. Couder, Phys. Rev. Lett. 68, 2098 (1992).

[7] L. Shang, Y. Cheng, and Y. Zhao, Chem. Rev. 117, 7964 (2017).

[8] A. Dinsmore, M. F. Hsu, M. Nikolaides, M. Marquez, A. Bausch, and D. Weitz, Science 298, 1006 (2002).

[9] W. Mickelson, S. Aloni, W.-Q. Han, J. Cumings, and A. Zettl, Science 300, 467 (2003).

[10] A. N. Khlobystov, D. A. Britz, A. Ardavan, and G. A. D. Briggs, Phys. Rev. Lett. 92, 245507 (2004).

[11] M. Tymczenko, L. F. Marsal, T. Trifonov, I. Rodriguez, F. Ramiro-Manzano, J. Pallares, A. Rodriguez, R. Alcubilla, and F. Meseguer, Adv. Mater. 20, 2315 (2008).

[12] S. A. Vanapalli, C. R. Iacovella, K. E. Sung, D. Mukhija, J. M. Millunchick, M. A. Burns, S. C. Glotzer, and M. J. Solomon, Langmuir 24, 3661 (2008).

[13] V. T. Cong, E.-O. Ganbold, J. K. Saha, J. Jang, J. Min, J. Choo, S. Kim, N. W. Song, S. J. Son, S. B. Lee et al., J. Am. Chem. Soc. 136, 3833 (2014).

[14] S. Sanwaria, A. Horechyy, D. Wolf, C.-Y. Chu, H.-L. Chen, P. Formanek, M. Stamm, R. Srivastava, and B. Nandan, Angew. Chem. Int. Ed. 53, 9090 (2014).

[15] L. Fu, C. Bian, C. W. Shields, D. F. Cruz, G. P. López, and P. Charbonneau, Soft Matter 13, 3296 (2017).

[16] T. Lee, K. Gizynski, and B. A. Grzybowski, Adv. Mater. 29, 1704274 (2017).

[17] J. H. Moon, S. Kim, G.-R. Yi, Y.-H. Lee, and S.-M. Yang, Langmuir 20, 2033 (2004).

[18] F. Li, X. Badel, J. Linnros, and J. B. Wiley, J. Am. Chem. Soc. 127, 3268 (2005).
[19] R. Liang, J. Xu, R. Deng, K. Wang, S. Liu, J. Li, and J. Zhu, ACS Macro Lett. 3, 486 (2014).

[20] Z. Chen, S. Ye, S. D. Evans, Y. Ge, Z. Zhu, Y. Tu, and X. Yang, Small 14, 1704015 (2018).

[21] R. O. Erickson, Science 181, 705 (1973).

[22] G. T. Pickett, M. Gross, and H. Okuyama, Phys. Rev. Lett. 85, 3652 (2000).

[23] H.-K. Chan, Phys. Rev. E 84, 050302(R) (2011).

[24] A. Mughal, H.-K. Chan, and D. Weaire, Phys. Rev. Lett. 106, 115704 (2011).

[25] A. Mughal, H.-K. Chan, D. Weaire, and S. Hutzler, Phys. Rev. E 85, 051305 (2012).

[26] H.-K. Chan, Philos. Mag. 93, 4057 (2013).

[27] M.Z. Yamchi and R. K. Bowles, Phys. Rev. Lett. 115, 025702 (2015).

[28] L. Fu, W. Steinhardt, H. Zhao, J. E. Socolar, and P. Charbonneau, Soft Matter 12, 2505 (2016).

[29] J. J. Crassous, A. M. Mihut, E. Wernersson, P. Pfleiderer, J. Vermant, P. Linse, and P. Schurtenberger, Nat. Commun. 5, 5516 (2014).

[30] A. A. Shah, B. Schultz, W. Zhang, S. C. Glotzer, and M. J. Solomon, Nat. Mater. 14, 117 (2015).

[31] E. Basurto, C. Haro-Pérez, C. A. Vargas, and G. Odriozola, Phys. Chem. Chem. Phys. 20, 27490 (2018).

[32] D. Frenkel, B. M. Mulder, and J. P. McTague, Phys. Rev. Lett. 52, 287 (1984).

[33] D. Frenkel and B. M. Mulder, Mol. Phys. 55, 1171 (1985).

[34] M. P. Allen and C. P. Mason, Mol. Phys. 86, 467 (1995).

[35] P. J. Camp, C. P. Mason, M. P. Allen, A. A. Khare, and D. A. Kofke, J. Chem. Phys. 105, 2837 (1996).

[36] G. Odriozola, J. Chem. Phys. 136, 134505 (2012).

[37] G. Bautista-Carbajal, A. Moncho-Jordá, and G. Odriozola, J. Chem. Phys. 138, 064501 (2013).

[38] A. Donev, F. H. Stillinger, P. M. Chaikin, and S. Torquato, Phys. Rev. Lett. 92, 255506 (2004).

[39] W. Jin, Y. Jiao, L. Liu, Y. Yuan, and S. Li, Phys. Rev. E 95, 033003 (2017).

[40] S. Torquato and Y. Jiao, Nature (London) 460, 876 (2009).

[41] See Supplemental Material at http://link.aps.org/ supplemental/10.1103/PhysRevLett.124.248002, which includes Refs. [20,40,42], for detailed analytic expressions of the packing densities of uni-zigzag structures and for the computational method used in this study.

[42] J. W. Perram and M. S. Wertheim, J. Comput. Phys. 58, 409 (1985).

[43] H.-K. Chan, Y. Wang, and H. Han, AIP Adv. 9, 125118 (2019).

[44] A. Mughal, Philos. Mag. 93, 4070 (2013).

[45] A. A. Shah, H. Kang, K. L. Kohlstedt, K. H. Ahn, S. C. Glotzer, C. W. Monroe, and M. J. Solomon, Small 8, 1551 (2012).

[46] M. Ganesan and M. J. Solomon, Soft Matter 13, 3768 (2017). 